\section{Methotrexate-associated toxicity in children with Down syndrome and acute lymphoblastic leukemia during consolidation therapy with high dose methotrexate according to ALL-BFM treatment regimen}

\author{
Mirko Kroll, ${ }^{1}$ Kirsten Kaupat-Bleckmann, ${ }^{1}$ Anja Mörickel, ${ }^{1}$ Julia Altenl, ${ }^{1}$ \\ Denis M. Schewel, ${ }^{1}$ Martin Stanullal, ${ }^{2}$ Martin Zimmermann, ${ }^{2}$ \\ Martin Schrappe ${ }^{1}$ and Gunnar Cario ${ }^{1}$ \\ ${ }^{1}$ Department of Pediatrics I, University Hospital Schleswig-Holstein, Kiel and \\ ${ }^{2}$ Department of Pediatric Hematology and Oncology, Hannover Medical School, \\ Hannover, Germany
}

\section{ABSTRACT}

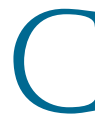
hildren with Down syndrome (DS) and acute lymphoblastic leukemia (ALL) often suffer from severe toxicities during treatment, especially with high-dose methotrexate (HD-MTX). Systematic data on methotrexate (MTX) toxicity in these patients are rare. We analyzed seven MTX-associated toxicities during consolidation therapy in 103 DS- and 1,109 non-DS-patients (NDS) with ALL (NDS-ALL) enrolled in ALL-Berlin-Frankfurt-Münster (ALL-BFM) trials between 1995-2016 and 1995-2007, respectively. Patients received four courses MTX (5 g/m² each) plus intrathecal MTX and 6-mercaptopurine (6-MP). From 2004 onwards, a dose of $0.5 \mathrm{~g} / \mathrm{m}^{2}$ in the first MTX course has been recommended for DSpatients. DS-patients showed higher rates of grade $3 / 4$ toxicities after the first course with $5 \mathrm{~g} / \mathrm{m}^{2}$ MTX compared to NDS-patients (grade 3/4 toxicities 62 in 45 DS-patients vs. 516 in 1,089 NDS-patients, $P<0.001)$. The dose reduction $\left(0.5 \mathrm{~g} / \mathrm{m}^{2}\right)$ in DS-patients has reduced toxicity (39 in 51 patients, $P<0.001$ ) without increasing the relapse risk (reduced dose, 5 -year cumulative relapse incidence $=0.09 \pm 0.04 v s$. high dose, $0.10 \pm 0.05, P=0.51$ ). MTX dose escalation to $1.0 \mathrm{~g} / \mathrm{m}^{2}$ for DS-patients who tolerated $0.5 \mathrm{~g} / \mathrm{m}^{2}$ $(n=28$ of 51 patients) did not result in an increased rate of grade $3 / 4$ toxicities after the second course $(P=0.285)$. Differences in MTX plasma levels at 42 and 48 hours after the start of the first methotrexate infusion did not explain higher toxicity rates in DS-patients treated with $0.5 \mathrm{~g} / \mathrm{m}^{2}$ compared to NDS-patients treated with $5 \mathrm{~g} / \mathrm{m}^{2}$. Within the DS cohort a higher MTX plasma level was associated with increased toxicity. In conclusion, dose reduction in the first MTX course reduced severe toxicities without increasing the risk of relapse. (ClinicalTrials.gov identifier: NTC00430118, NCT01117441).

\section{Introduction}

Children and adolescents with Down syndrome (DS) DS have an approximately 10-fold higher risk of acquiring acute lymphoblastic leukemia (ALL) ${ }^{1}$ and have shown an inferior outcome compared to children with NDS-ALL. ${ }^{2 \cdot 5}$ The worse outcome for DS-ALL has been attributed to a higher risk of relapse as well as higher levels of chemotherapy-associated toxicities and treatment-related mortality., 2,68 Severe toxicities may eventually lead to chemotherapy dose reduction which in turn might increase the risk of relapse..$^{2,5,6}$

Methotrexate (MTX is a folate antagonist that interferes with the de novo synthesis of nucleotides in proliferating cells and plays a crucial role in the treatment of pediatric ALL in which MTX is administered in high doses (HD-MTX, $\geq 0.5 \mathrm{~g} / \mathrm{m}^{2}$ per intravenous [i.v.] application). ${ }^{9}$ Many patients with DS-ALL suffer from severe side effects after receiving HD-MTX. Common MTX-associated side effects in DSALL are severe mucositis and stomatitis, infections, bone marrow suppression, hepato-, nephro- and neurotoxicity., ${ }^{2,6-8}$

In order to extend our knowledge on MTX-associated toxicities in DS-ALL, we
Haematologica 2020

Volume 105(4):1013-1020

\section{Correspondence:}

GUNNAR CARIO

gunnar.cario@uksh.de

Received: April 15, 2019.

Accepted: July 24, 2019.

Pre-published: August 1, 2019.

doi:10.3324/haematol.2019.224774

Check the online version for the most updated information on this article, online supplements, and information on authorship \& disclosures: www.haematologica.org/content/105/4/1013

(C)2020 Ferrata Storti Foundation

Material published in Haematologica is covered by copyright. All rights are reserved to the Ferrata Storti Foundation. Use of published material is allowed under the following terms and conditions:

https://creativecommons.org/licenses/by-nc/4.0/legalcode. Copies of published material are allowed for personal or internal use. Sharing published material for non-commercial purposes is subject to the following conditions:

https://creativecommons. org/licenses/by-nc/4.0/legalcode, sect. 3. Reproducing and sharing published material for commercial purposes is not allowed without permission in writing from the publisher. 
analyzed clinical data from DS-ALL and NDS-ALL patients who were treated according to ALL-BFM protocols in Germany and Switzerland (ALL-BFM 1995, ALLBFM 2000 and AIEOP-BFM ALL 2009). We focused on toxicities occurring after HD-MTX administrations during the consolidation element Protocol $M$ in which patients receive four courses of i.v. HD-MTX $\left(5 \mathrm{~g} / \mathrm{m}^{2}\right.$ each, 24-hour infusion).

Additionally, we investigated the influence of the rs1051266 80G >A polymorphism of the chromosome 21encoded folate carrier SLC19A1 (RFC1) on MTX toxicity in DS-ALL. SLC19A1 functions as the main transporter for MTX into cells. Therefore rs1051266 may alter the transportation rate of MTX into cells and thus may affect the severity of toxicities. ${ }^{10-12}$

\section{Methods}

\section{Patients}

A total of 1,212 patients were selected from three consecutive multicenter ALL-BFM trials (ALL-BFM 95, ALL-BFM 2000 and AIEOP-BFM ALL 2009; diagnosed between January 13, 1996 and September 6, 2016 for DS-ALL and between April 11, 1995 and May 4, 2007 for NDS-ALL patients) according to the following inclusion criteria: age at diagnosis between $\geq 1$ year and $\leq 18$ years, no initial high risk (HR) features, consolidation treatment with HD-MTX (Protocol M) and availability of treatment and toxicity data. Detailed information on the different recruitment periods and treatment stratification is given in the Online Supplementary Materials and Methods. Informed consent from the patients and/or guardians was obtained and the studies were approved by the responsible ethical committees.

Consolidation Protocol M is an 8-week therapy element given to non-HR patients only. Patients receive four courses of i.v. HDMTX (5 g/m² each, 24-hour infusion) every second week in addition to an age-adapted intrathecal MTX and daily oral 6-MP (25 $\mathrm{mg} / \mathrm{m}^{2} /$ day). MTX plasma levels were measured at various time points after the start of each MTX infusion and each HD-MTX course was followed by an i.v. leucovorine (LCV) rescue $\left(15 \mathrm{mg} / \mathrm{m}^{2}\right.$ ) at 42, 48 and 54 hours after the start of the MTX infusion (for details on Protocol M see the Online Supplementary Material and Methods). ${ }^{13}$ Patients who qualified for the HR group only after the start of Protocol $\mathrm{M}$ and who were therefore allocated to the HR treatment arm of the respective study were included in the toxicity analysis for the HD-MTX courses they had received (usually only the first course) but not for outcome analysis.

As of 2004, the study group recommended to administer the first HD-MTX course with a reduced dose of $0.5 \mathrm{~g} / \mathrm{m}^{2}$ in order to reduce severe toxicities in DS-ALL patients. A subsequent increase of MTX dosages could be performed if no severe toxicity had occurred. After each HD-MTX course, toxicity grading was performed for leukopenia, thrombocytopenia, infections, stomatitis, hepatotoxicity, nephrotoxicity and bilirubinemia according to the Common Terminology Criteria for Adverse Events (CTCAE) version 2.0 (for details see the Online Supplementary Materials and Methods and Online Supplementary Table S1). In some patients reporting was incomplete covering only some of the seven toxicities.

\section{Statistical analysis}

Statistical analysis included $\chi^{2}$-, Fisher's exact, Mann-Whitney$\mathrm{U}$ and $\mathrm{McNemar}$ tests as indicated in the figure legends and tables. The 5-year-cumulative incidence risk of relapse was calculated according to Kalbfleisch and Prentice and compared using the Gray's test. Analyses were done using IBM SPSS 22 and SAS 9.4.
A $P$-value $\leq 0.05$ was considered to be statistically significant.

\section{DNA and allelic discrimination assay}

Information on patient DNA preparation and SLC19A1 rs1051266 80G>A genotyping can be found in the Online Supplementary Materials and Methods.

\section{Results}

Patients, treatment discontinuation and MTX dosages

1,212 patients were included in this study, 103 DS-ALL and 1,109 NDS-ALL. The comparison of both groups showed no significant differences in the median age at diagnosis, sex distribution, and white blood cell counts at diagnosis (Table 1). None of the DS patients had T-cell ALL in contrast to $12.4 \%$ of the NDS-ALL group ( $P=0.006)$ and a hyperdiploid karyotype was less frequent in DSALL compared to NDS-ALL patients $(P=0.002)$. Furthermore, NDS-ALL had more ETV6-RUNX1 rearrangements compared to DS-ALL patients $(P<0.001)$ (Table 1).

Of the 103 DS-ALL patients who started HD-MTX consolidation 95 patients $(92.2 \%)$ completed the protocol. Seven DS-ALL patients discontinued consolidation treatment at various time points due to a switch to HR treatment $(n=4)$ or severe infections $(n=2)$. Analysis could not be performed in one patient with DS-ALL for the fourth HD-MTX course due to incomplete toxicity data. One DS-ALL patient died after the second course from septic shock in neutropenia after receiving $5 \mathrm{~g} / \mathrm{m}^{2}$ MTX doses in the first and second course. In contrast, 1,083 of 1,109 NDS-ALL patients $(97.7 \%)$ completed consolidation. Consolidation discontinuation in 26 NDS-ALL patients was due to a switch to HR treatment $(n=24)$ or severe toxicities (neurotoxicity, $\mathrm{n}=1$ and severe infection, $\mathrm{n}=1$ ). Three additional patients could not be analyzed due to incomplete data. None of the NDS-ALL patients died during HD-MTX consolidation (Figure 1).

As of 2004, the ALL-BFM study group recommended for DS-ALL patients to administer a reduced MTX dose of 0.5 $\mathrm{g} / \mathrm{m}^{2}$ in the first HD-MTX course and to subsequently increase the dose if no severe side effects occur. Therefore, 51 of 103 (49.5\%) DS-ALL patients received the first HDMTX course with a dose of $0.5 \mathrm{~g} / \mathrm{m}^{2}( \pm 10 \%)$ and 45 of 103 $(43.7 \%)$ received $5 \mathrm{~g} / \mathrm{m}^{2}( \pm 10 \%)$ in the first course (Table 1). Of note, in some DS-ALL patients a MTX dose of 0.5 $\mathrm{g} / \mathrm{m}^{2}$ was given already before 2004 and some DS-ALL patients still received $5 \mathrm{~g} / \mathrm{m}^{2}$ doses after the 2004 recommendation, (DS-ALL with $0.5 \mathrm{~g} / \mathrm{m}^{2}$ MTX in the first course before $2004, \mathrm{n}=6$ of $42[14.3 \%]$ and $5.0 \mathrm{~g} / \mathrm{m}^{2}$ in first course since $2004, n=11$ of $61[18.0 \%])$.

In contrast, 1,089 of $1,109(98.2 \%)$ non-DS-ALL patients received $5 \mathrm{~g} / \mathrm{m}^{2}$ MTX. As expected, the MTX dosages in the course of the consolidation treatment were more heterogeneous in DS-ALL as compared to NDS-ALL (Table 1).

\section{Toxicities after HD-MTX}

Initially we focused on toxicities after the first HD-MTX course because latter courses might be more biased by toxicities and MTX dosages from previous HD-MTX courses, especially in those patients for whom the dose has been adopted after the recommendation in 2004. After receiving a MTX dose of $5 \mathrm{~g} / \mathrm{m}^{2}$ patients with DS-ALL showed significantly higher rates of grade $3 / 4$ leukopenia, thrombocytopenia, infections and stomatitis compared to 
Table 1. Patient characteristics, methotrexate dosages in the first high-dose methotrexate course and total methotrexate dosage trend during consolidation for Down syndrome and non-Down syndrome acute lymphoblastic leukemia patients.

\begin{tabular}{|c|c|c|c|c|}
\hline & & $\begin{array}{c}\text { DS-ALL } \\
(n=103)\end{array}$ & $\begin{array}{l}\text { NDS-ALL } \\
(n=1,109)\end{array}$ & $P$ \\
\hline Age at diagnosis & Median & 4.8 years & 5.9 years & $0.564^{\mathrm{a}}$ \\
\hline Range & & $1.2-17.7$ years & $1.0-18.0$ years & \\
\hline Sex & Male & 64/103 (62.1\%) & $599 / 1,109$ (54.0\%) & $0.113^{b}$ \\
\hline Female & & 39/103 (37.9\%) & $510 / 1,109(46.0 \%)$ & \\
\hline WBC at diagnosis & Median & $9,850 / \mu \mathrm{L}$ & $10,700 / \mu \mathrm{L}$ & $0.411^{\mathrm{a}}$ \\
\hline Range & & $870-205,000$ & $100-686,000$ & \\
\hline$<10,000 / \mu \mathrm{L}$ & & $55 / 102(53.9 \%)$ & $544 / 1,109$ (49.1\%) & \\
\hline $10,000-49,999 / \mu \mathrm{L}$ & & 37/102 (36.3\%) & $364 / 1,109$ (32.8\%) & \\
\hline$>50,000 / \mu \mathrm{L}$ & & 10/102 (9.8\%) & 201/1,109 (18.1\%) & \\
\hline B-/T-cell lineage & B-ALL & $101 / 101(100 \%)$ & $937 / 1,070(87.6 \%)$ & $<0.001^{\text {b }}$ \\
\hline T-ALL & & 0/101 (0\%) & $133 / 1,070(12.4 \%)$ & \\
\hline \multirow[t]{2}{*}{ Karyotype } & Normal karyotype & $28 / 55$ (50.9\%) & $61 / 215(28.4 \%)$ & $0.002^{\mathrm{b}}$ \\
\hline & Hyperploidy (> 50 chr.) & $5 / 55(9.1 \%)$ & $62 / 215(28.8 \%)$ & $0.002^{\mathrm{b}}$ \\
\hline \multirow[t]{2}{*}{ Genetic features } & ETV6-RUNX1 & 7/92 (7.6\%) & $205 / 810(25.3 \%)$ & $<0.001^{\mathrm{b}}$ \\
\hline & BCR-ABL & 0/92 (0\%) & $0 / 810(0 \%)$ & \\
\hline \multirow[t]{3}{*}{ MTX dose $1^{\text {st }}$ course } & $5 \mathrm{~g} / \mathrm{m}^{2} \pm 10 \%$ (full dose) & 45/103 (43.7\%) & 1,089/1,109 (98.2\%) & \\
\hline & $0.551-4.499 \mathrm{~g} / \mathrm{m}^{2}$ & $7 / 103$ (6.8\%) & 20/1,109 (1.8\%) & \\
\hline & $0.5 \mathrm{~g} / \mathrm{m}^{2} \pm 10 \%$ (reduced dose, low dose) & $51 / 103(49.5 \%)$ & 0/1,109 (0\%) & \\
\hline \multirow[t]{9}{*}{ MTX dosage trend ${ }^{c}$} & started with full dose, full dose continued & 25/103 (24.3\%) & $1,049 / 1,109(94.6 \%)$ & \\
\hline & started with full dose, then reduced & $14 / 103(13.6 \%)$ & 12/1,109 (1.1\%) & \\
\hline & started with low dose, then escalated & $31 / 103(30.1 \%)$ & $0 / 1,109(0 \%)$ & \\
\hline & started with low dose, low dose continued & 19/103 (18.4\%) & $0 / 1,109(0 \%)$ & \\
\hline & discontinuation due to death & $1 / 103(1.0 \%)$ & 0/1,109 (0\%) & \\
\hline & discontinuation due to severe toxicity & 2/103 (1.9\%) & $2 / 1,109(0.2 \%)$ & \\
\hline & intermediate dose in all four courses ${ }^{\text {de }}$ & 6/103 (5.8\%) & $19 / 1,109(1.7 \%)$ & \\
\hline & switch to high risk treatmente,f & 4/103 (3.9\%) & $24 / 1,109(2.2 \%)$ & \\
\hline & incomplete data ${ }^{\mathrm{e}, \mathrm{d}}$ & $1 / 103(1.0 \%)$ & $3 / 1,109(0.3 \%)$ & \\
\hline
\end{tabular}

ALL: acute lymphoblastic leukemia; DS-ALL: Down syndrome ALL; NDS-ALL: non-Down syndrome ALL; chr; chromosomes; y: years; MTX: methotrexate; HD; high dose; n. a.: not applicable; WBC: white blood cell count; ${ }^{a}$ Mann-Whitney U test; ${ }^{\mathrm{b}} \chi^{2}$ test; ${ }^{\mathrm{c}}$ overall changes in MTX dosage in the course of consolidation therapy; ${ }^{\mathrm{d}}$ one DS-ALL patient received three courses with intermediate MTX doses but no information about the last course were available; 'one NDS-ALL patient was allocated to high risk treatment after receiving three courses of intermediate MTX doses during consolidation; 'patients that started HD-MTX consolidation but qualified for high risk treatment during Protocol M.

NDS-ALL who received the same dose (leukopenia, DSALL $\mathrm{n}=19$ of $44[43.2 \%]$ vs. NDS-ALL $\mathrm{n}=255$ of 961 [26.5\%], $P=0.023$; thrombocytopenia, 14 of 43 [32.6\%] vs. 84 of 957 [8.8\%], $P<0.001$; infections, 4 of $43[9.3 \%]$ vs. 15 of 939 [1.6\%], $P=0.008$; stomatitis, 18 of 43 [41.9\%] vs. 56 of 951 [5.9\%], $P<0.001$ ) (Figure 2A). An initial MTX dose of $0.5 \mathrm{~g} / \mathrm{m}^{2}$ led to a significantly reduced rate of grade $3 / 4$ leukopenia, thrombocytopenia and stomatitis in DSALL compared to DS-ALL who received $5 \mathrm{~g} / \mathrm{m}^{2}$ (leukopenia, $0.5 \mathrm{~g} / \mathrm{m}^{2} \mathrm{n}=11$ of 50 [22.0\%] vs. $5 \mathrm{~g} / \mathrm{m}^{2} \mathrm{n}=19$ of 44 $[43.2 \%], P=0.045$; thrombocytopenia, 7 of $50[14.0 \%]$ vs. 14 of 43 [32.6\%], $P=0.046$; stomatitis, 9 of 49 [18.4\%] vs. 18 of 43 [41.9\%], $P=0.021)$. However, DS-ALL patients treated with a reduced MTX dose still suffered from significantly higher rates of grade $3 / 4$ infections, stomatitis and hepatotoxicity compared to NDS-ALL who received 5 $\mathrm{g} / \mathrm{m}^{2}$ MTX (infections, DS-ALL $\mathrm{n}=3$ of 48 [6.3\%] vs. NDSALL $\mathrm{n}=15$ of 939 [1.6\%], $P=0.05$; stomatitis, 9 of 49 [18.4\%] vs. 56 of 951 [5.9\%], $P=0.003$; hepatotoxicity, 9 of 49 [18.4\%] vs. 59 of 945 [6.2\%], $P=0.004)$. An increase of MTX after the first dose to a median dose of $1.0 \mathrm{~g} / \mathrm{m}^{2}$ (range $0.94-2.06 \mathrm{~g} / \mathrm{m}^{2}$ ) in the second course was feasible in 28 of $51(54.9 \%)$ DS-ALL patients who initially received $0.5 \mathrm{~g} / \mathrm{m}^{2}$ (Figure 2B). Dose escalation did not result in an increased rate of toxicities (11 grade $3 / 4$ toxicities in 28 patients after the first course with $0.5 \mathrm{~g} / \mathrm{m}^{2}$ MTX vs. 16 of 28 patients after the second course with $1.0 \mathrm{~g} / \mathrm{m}^{2}$ MTX, $P=0.285)$.

Further dose escalation in subsequent HD-MTX courses occurred in 15 of these 28 dose escalated patients: 12 patients $(23.5 \%$ of the initial 51 DS-ALL patients with 0.5 $\mathrm{g} / \mathrm{m}^{2}$ MTX in the first course) received $5 \mathrm{~g} / \mathrm{m}^{2}$ from the third course on and three additional patients in the fourth course. In the remaining 13 of the 28 patients, an intermediate MTX dose was either continued throughout the remaining consolidation $(n=10)$, or was reduced to a low dose in the fourth course $(n=1)$, or was decreased to an intermediate dose in the fourth course after being increased to $5 \mathrm{~g} / \mathrm{m}^{2}$ in the third course $(\mathrm{n}=1)$ or the therapy was switched to HR treatment after the second course $(n=1)$. In 4 of 51 patients who were initially treated with $0.5 \mathrm{~g} / \mathrm{m}^{2}$ MTX in the first course, MTX dose escalation 


\section{Down syndrome-ALL}

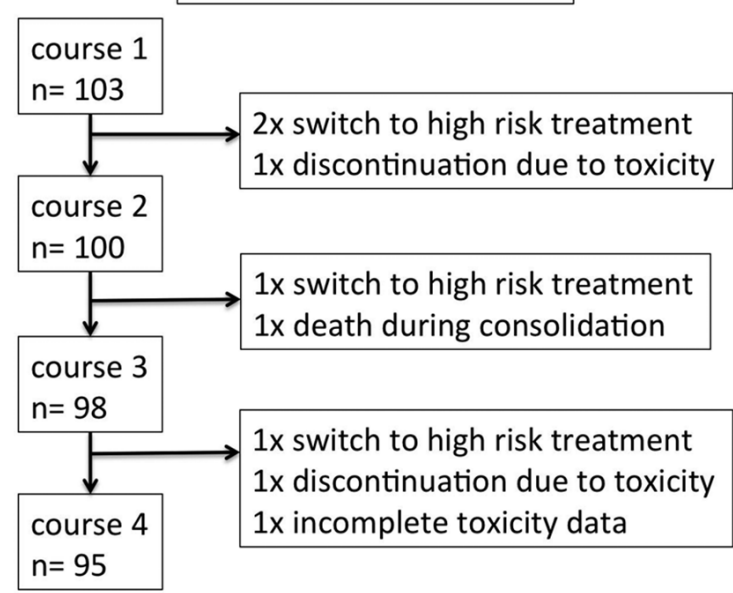

Non-Down syndrome-ALL

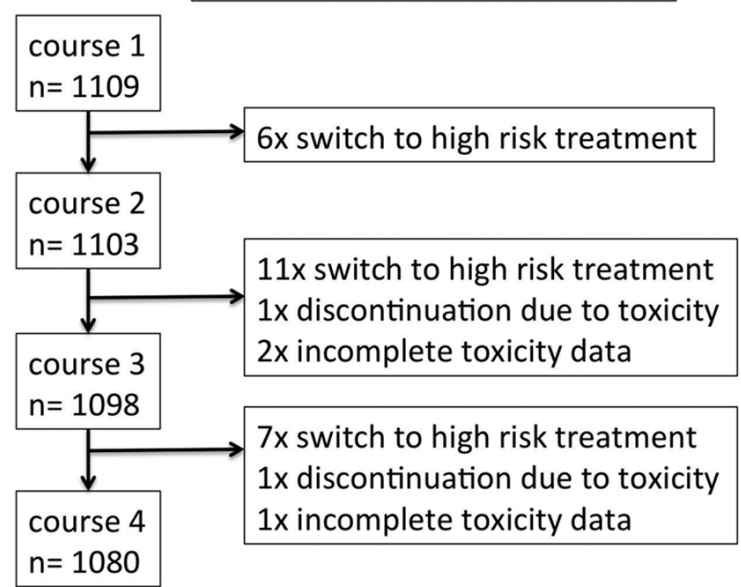

Figure 1. Flow chart showing patient numbers in individual high-dose methotrexate courses including drop outs during high dose methotrexate consolidation.

was performed later in the course of consolidation, i.e. in the third $(n=3)$ or the fourth course $(n=1)$. The remaining 19 of the 51 patients were treated with a $0.5 \mathrm{~g} / \mathrm{m}^{2}$ MTX dose throughout the whole consolidation phase.

Next we analyzed toxicities after the first and the forth HD-MTX course of all the DS-ALL patients who completed consolidation ( $n=95$ ) (Figure 2C-D). DS-ALL patients showed a significant decrease in the rate of grade $3 / 4$ stomatitis after the last course as compared to the initial course (stomatitis, in the first course $n=27$ of 93 [29.0\%], in the fourth course $\mathrm{n}=9$ of 91 [9.9\%], $P=0.002)$. In contrast, the number of patients with grade $3 / 4$ leukopenia significantly increased (30 of 94 [31.9\%] vs. 45 of 89 [50.6\%], $P=0.01)$. Similarly, NDS-ALL patients showed a decrease in grade $3 / 4$ stomatitis and an increase in grade $3 / 4$ leukopenia during the course of consolidation therapy (stomatitis, 53 of 942 [5.6\%] vs. 9 of 923 [1.0\%], $P<0.001$; leukopenia, 250 of 952 [26.3\%] vs. 477 of 935 [51.0\%], $P<0.001)$.

\section{Impact of MTX dose reduction on cumulative incidence of relapses}

The 5-year-cumulative incidence risk of relapse (5y-CIR) was compared between DS-ALL patients who received a dose of $0.5 \mathrm{~g} / \mathrm{m}^{2}$ in the first HD-MTX course with those who received $5 \mathrm{~g} / \mathrm{m}^{2}$ (Figure $3 \mathrm{~A}$ ). No significant differences in $5 \mathrm{y}$-CIR were observed $\left(0.5 \mathrm{~g} / \mathrm{m}^{2}\right.$ subgroup $\mathrm{n}=5$ of $50,5 y-C I R \pm S E=0.09 \pm 0.04$ vs. $5 \mathrm{~g} / \mathrm{m}^{2}$ subgroup $\mathrm{n}=7$ of 41 , $5 y-C I R=0.10 \pm 0.05, P=0.51)$. Next we compared the $5 y-$ CIR of DS-ALL patients who received a $0.5 \mathrm{~g} / \mathrm{m}^{2}$ MTX dose in the first course and increased dosages in subsequent courses with those of patients who were treated with a MTX dose of $0.5 \mathrm{~g} / \mathrm{m}^{2}$ throughout the whole consolidation phase. No significant CIR difference between both groups was observed $\left(0.5 \mathrm{~g} / \mathrm{m}^{2}\right.$ and escalated, $5 \mathrm{y}-\mathrm{CIR}=0.14 \pm 0.07$ vs. $0.5 \mathrm{~g} / \mathrm{m}^{2}$ and continued, $5 y-C I R=0.00 \pm 0.00, P=0.42$ ) (Figure $3 B$ ).

\section{Association of MTX plasma levels and toxicity}

To investigate whether differences in MTX plasma levels might explain the high rates of severe toxicities observed in DS-ALL we analyzed MTX plasma levels of all patients at 42 and 48 hours after the start of the initial MTX infusion (Figure 4A-B). At 42 hours median MTX plasma levels in DS-ALL were $0.21 \mu \mathrm{mol} / \mathrm{L}$ (range 0.05 $3.40 \mu \mathrm{mol} / \mathrm{L})$ for $0.5 \mathrm{~g} / \mathrm{m}^{2} \mathrm{MTX}$ and $0.90 \mu \mathrm{mol} / \mathrm{L}(0.17$ 4.60) for $5 \mathrm{~g} / \mathrm{m}^{2}$ MTX $(P<0.001)$. At 48 hours DS-ALL median plasma levels were $0.16 \mu \mathrm{mol} / \mathrm{L}(0.01-1.80)$ and $0.43 \mu \mathrm{mol} / \mathrm{L}(0.22-3.60)$, respectively $(P<0.001)$. For NDSALL patients who received a $5 \mathrm{~g} / \mathrm{m}^{2}$ MTX dose median MTX plasma levels were $0.50 \mu \mathrm{mol} / \mathrm{L}(0.04-21.60)$ at 42 hours and $0.31 \mu \mathrm{mol} / \mathrm{L}(0.00-10.99)$ at 48 hours after the start of the first MTX infusion. Thus, at both time points DS-ALL patients showed significantly higher MTX plasma levels after $5 \mathrm{~g} / \mathrm{m}^{2}$ MTX compared to NDS-ALL who received the same dose. DS-ALL patients treated with 0.5 $\mathrm{g} / \mathrm{m}^{2}$ MTX had significantly lower MTX plasma levels compared to DS-ALL treated with $5 \mathrm{~g} / \mathrm{m}^{2}$ MTX and NDSALL treated with $5 \mathrm{~g} / \mathrm{m}^{2}$ at both time points $(P<0.001$ for each comparison). To further analyze whether MTX plasma levels may have an impact on toxicity within the DSALL cohort we analyzed the occurrence of grade 3/4 toxicities according to MTX plasma level quartiles at 42 and 48 hours (Figure 4C-D). At 42 hours DS-ALL patients with MTX plasma levels within the highest quartile $(\geq 0.905$ $\mu \mathrm{mol} / \mathrm{L}, \mathrm{n}=23$ ) suffered from grade $3 / 4$ leukopenia, thrombocytopenia and stomatitis significantly more often compared to patients within the lowest quartile $(\leq 0.200$ $\mu \mathrm{mol} / \mathrm{L}, \mathrm{n}=24$ ) (leukopenia, highest quartile $\mathrm{n}=7$ of 22 [31.8\%] vs. lowest quartile $\mathrm{n}=1$ of 22 [4.6\%], $P=0.046$; thrombocytopenia, 7 of $22[31.8 \%]$ vs. 0 of $22[0 \%]$, $P=0.009$; stomatitis, 9 of $21[42.9 \%$ vs. 0 of $22[0 \%]$, $P=0.001)$. DS-ALL patients with MTX levels within the highest quartile at 48 hours $(\geq 0.470 \mu \mathrm{mol} / \mathrm{L}, \mathrm{n}=22)$ showed significantly higher rates of grade $3 / 4$ thrombocytopenia and stomatitis compared to the lowest quartile $(\leq 0.160 \mu \mathrm{mol} / \mathrm{L}, \mathrm{n}=25)$ (thrombocytopenia, 9 of 21 [42.9\%] vs. 1 of 25 [4.0\%], $P=0.003$; stomatitis, 10 of 20 $[50.0 \%]$ vs. 2 of $25[8.0 \%], P=0.002)$.

\section{SLC19A1 polymorphism rs1051266}

The chromosome 21-encoded and ubiquitously 
A

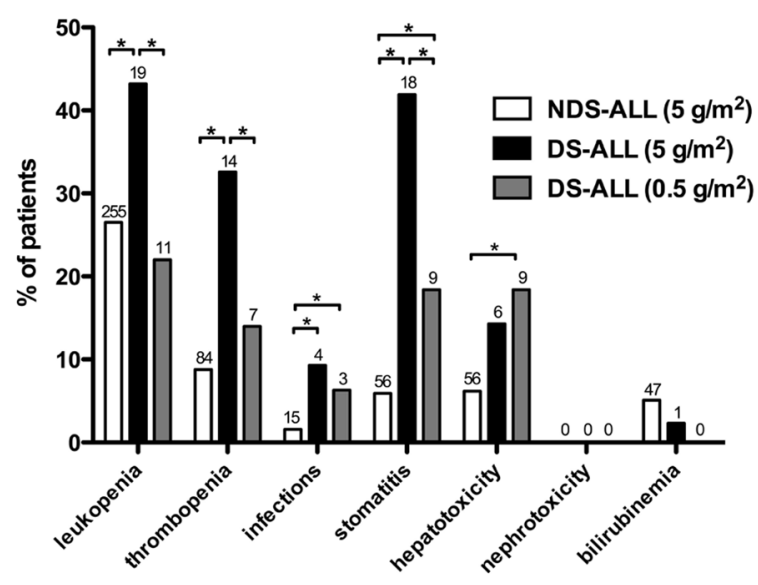

C

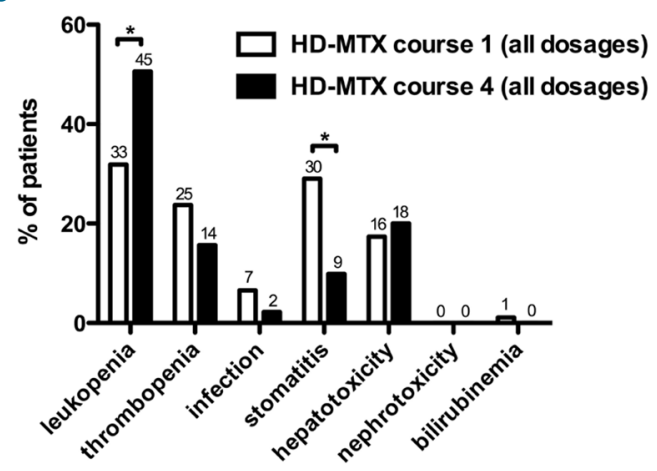

B

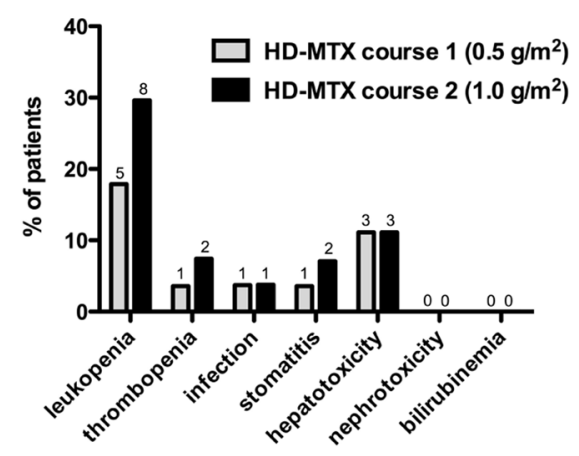

D

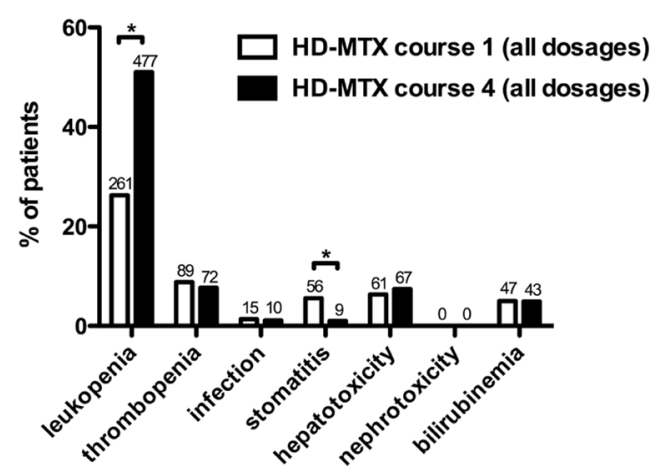

Figure 2. Comparison of toxicities after first high-dose methotrexate (HD-MTX) course in Down syndrome acute lymphoblastic leukemia (DS-ALL) versus non-Down syndrome acute lymphoblastic leukemia (NDS-ALL) and comparison of toxicities in DS-ALL after the initial and later HD-MTX courses. (A) Comparison of grade 3/4 toxicities after application of the first HD-MTX course in DS-ALL patients who received $5 \mathrm{~g} / \mathrm{m}^{2}(\mathrm{n}=45$ of 103$)$ or $0.5 \mathrm{~g} / \mathrm{m}^{2} \mathrm{methotrexate}(\mathrm{MTX})(\mathrm{n}=51$ of 103 ) and NDSALL patients who received $5 \mathrm{~g} / \mathrm{m}^{2}$ MTX ( $\left.\mathrm{n}=1,089 / 1,109\right) . * P \leq 0.05$, Fisher's exact test. (B) Comparison of grade $3 / 4$ toxicities after the first and second HD-MTX course in DS-ALL patients who initially received $0.5 \mathrm{~g} / \mathrm{m}^{2}$ MTX and were escalated to a median MTX dose of $1.0 \mathrm{~g} / \mathrm{m}^{2}$ in the second course ( $\mathrm{n}=28$ of 51 ). No significant differences according to McNemar test. (C) and (D) Comparison of grade 3/4 toxicities after the first and last HD-MTX course in all DS-ALL (C) and NDS-ALL patients (D), including patients with intermediate MTX doses. ${ }^{*} P \leq 0.05$ according to McNemar-test. The number on top of each bar represents the number of patients.

expressed folate carrier SLC19A1 (RFC1) functions as the main MTX transporter into cells. ${ }^{10}$ The single nucleotide polymorphism (SNP) rs1051266 80G>A has been described to affect the transportation rate of the SLC19A1 carrier. ${ }^{11}$ To gain further insight into MTX-associated toxicities in DS-ALL patients, we investigated whether rs1051266 allele combinations were associated with higher rates of toxicity in DS-ALL patients. Therefore, we genotyped rs1051266 80G>A in $\mathrm{n}=95$ of 103 DS-ALL patients and compared grade $3 / 4$ toxicities after the initial HD-MTX course between the homozygous allele carriers (i.e. GGG and AAA) (Online Supplementary Table S2). After subgrouping according to the administered MTX dose, no statistically significant association between the occurrence of grade $3 / 4$ toxicities and the allele status was observed, except for a significant higher rate of grade $3 / 4$ leukopenia after the first HD-MTX course in GGG allele carriers who received $5 \mathrm{~g} / \mathrm{m}^{2}$ MTX compared to AAA carriers that received the same dose (GGG $\mathrm{n}=8$ of 9 [88.9\%] vs. AAA $\mathrm{n}=0$ of $5[0 \%], P=0.003)$. However, this finding could not be reproduced for subsequent courses. Moreover, no significant correlation between other allele combinations and toxicities as well as allele carrier status and median MTX plasma levels at 42 and $48 \mathrm{~h}$ after the start of the initial MTX infusion were observed.

\section{Discussion}

In order to increase our knowledge of MTX-associated toxicities in DS-ALL patients after HD-MTX administration, we analyzed treatment and toxicity data of a large DS-ALL cohort uniformly treated according to ALL-BFM protocols. DS-ALL patient characteristics differed slightly from those in published studies, ${ }^{2,6,14}$ most likely because only patients who underwent HD-MTX consolidation treatment in the non-HR arm were included.

MTX dosages in DS-ALL were heterogeneous, especially since the release of the 2004 study group recommendations of a MTX starting dose of $0.5 \mathrm{~g} / \mathrm{m}^{2}$ for the first course which can eventually be increased in the following courses in the absence of severe toxicity. MTX dose heterogeneity and higher levels of MTX-associated toxicities have already been described by us and others. $2,5,8,15,16$ 
A

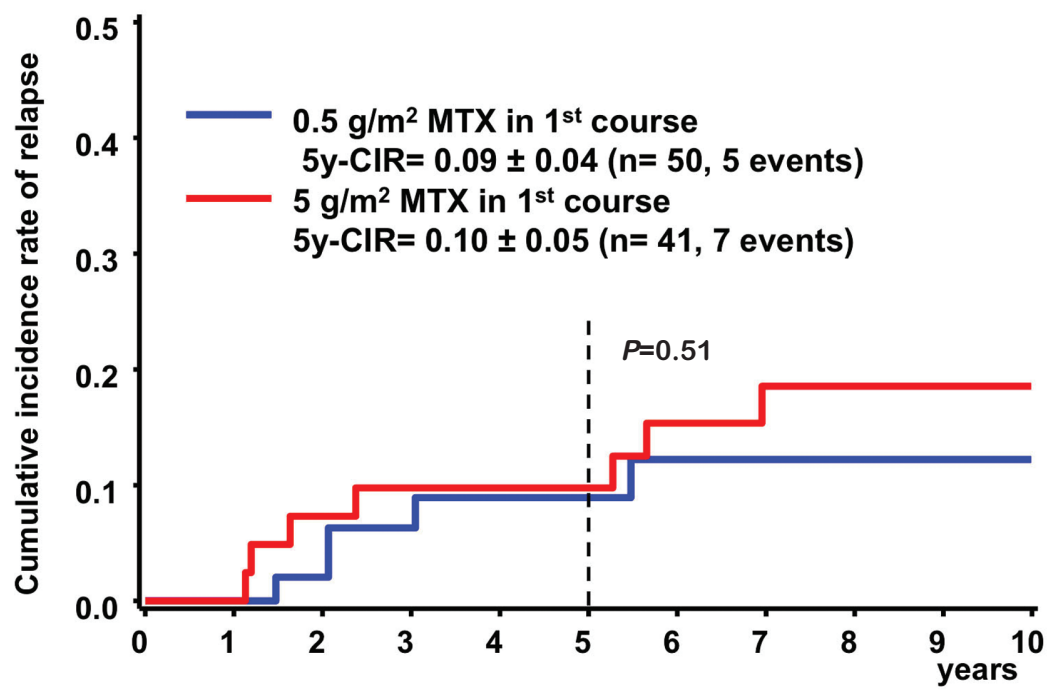

B

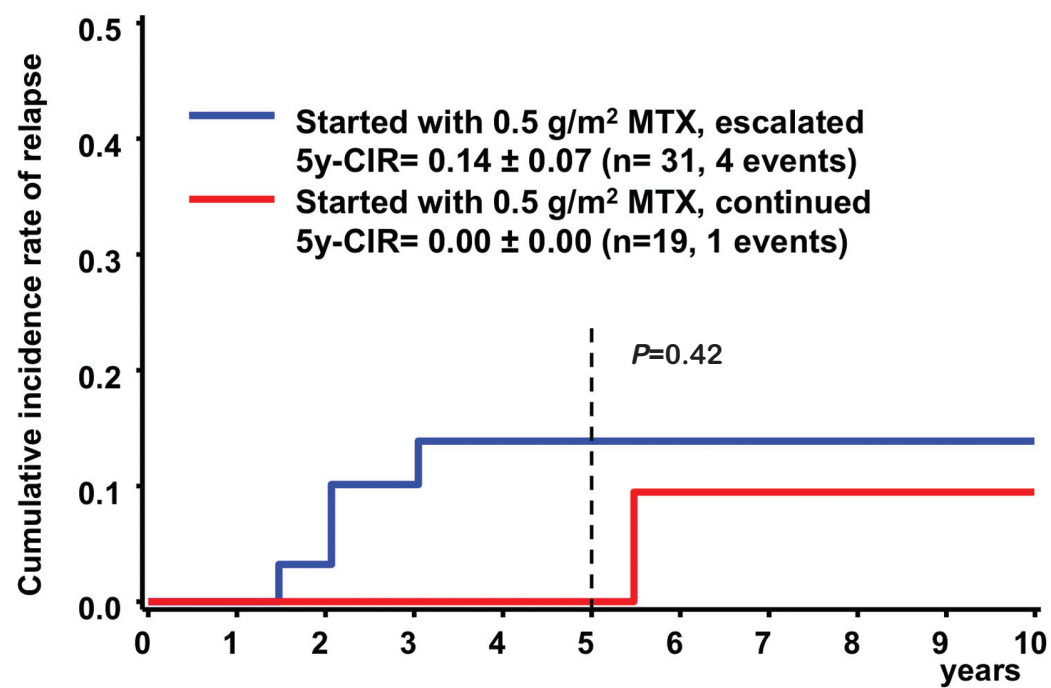

Figure 3. Five-year-cumulative incidence risk of relapse in Down syndrome acute lymphoblastic leukemia. (A) Comparison of the 5-year-cumulative incidence risk (5y-CIR) of Down syndrome acute lymphoblastic leukemia (DS-ALL) patients who received a first high dose methotrexate (HD-MTX) course of $0.5 \mathrm{~g} / \mathrm{m}^{2}$ (blue) or $5 \mathrm{~g} / \mathrm{m}^{2}$ MTX (red). No significant differences according to Gray's test. (B) Comparison of the $5 y$-CIR of DS-ALL who initially received $0.5 \mathrm{~g} / \mathrm{m}^{2}$ MTX in the first course and were eventually dose escalated in the course of consolidation with DSALL who received $0.5 \mathrm{~g} / \mathrm{m}^{2}$ MTX throughout the whole consolidation therapy. No significant differences were found according to Gray's test.

Together, these findings point to an increased awareness of the higher susceptibility of DS-ALL patients to MTXrelated toxicity and the need of MTX dose modifications in recent years.

In DS-ALL patients who received a MTX dose of $5 \mathrm{~g} / \mathrm{m}^{2}$ in the first course we observed significantly higher rates of grade $3 / 4$ leukopenia, thrombocytopenia, infection and stomatitis compared to NDS-ALL. Similar results in smaller cohorts of heterogeneously treated DS-ALL patients have also been observed by other groups. ${ }^{5,8,15,16}$ However, here we showed for the first time that the administration of a low MTX dose of $0.5 \mathrm{~g} / \mathrm{m}^{2}$ to patients with DS-ALL leads to a significant reduction of grade $3 / 4$ toxicity. Furthermore, the MTX dose could subsequently be escalated to $1.0 \mathrm{~g} / \mathrm{m}^{2}$ in more than half of the patients without resulting in higher rates of grade $3 / 4$ toxicities after the second course compared to the initial course. These data support the study group's recommendation to increase the MTX dose if no severe toxicity occurrs in the previous course. However, since reduced doses still bear a risk of severe side effects in individual DS-ALL patients, consoli- dation treatment should be administered cautiously and under close clinical control.

Dose reduction of chemotherapeutic drugs during cancer treatment may raise the concern of impairing longterm outcomes in the affected patients. Here, we did not find any differences in the 5y-CIR in DS-ALL patients who started consolidation with $5 \mathrm{~g} / \mathrm{m}^{2}$ MTX compared to patients who received $0.5 \mathrm{~g} / \mathrm{m}^{2}$ MTX. These findings raise the question whether DS-ALL patients require a high MTX dose in consolidation or whether a low dose between $0.5-1.0 \mathrm{~g} / \mathrm{m}^{2}$ might be sufficient to optimize their outcome by balancing treatment-related complications with relapse rates. While others found impaired outcome of DS-ALL patients who were treated with reduced drug doses, ${ }^{2,5}$ two studies described no differences in event free and overall survival between patients with or without dose reductions. ${ }^{15,16}$ The authors speculate that modern, more effective ALL therapy and supportive treatment may contribute to an improved outcome in DS patients treated with a reduced dose. Other groups also described a trend towards a better outcome for DS-ALL in recent times. ${ }^{6,14}$ 
A

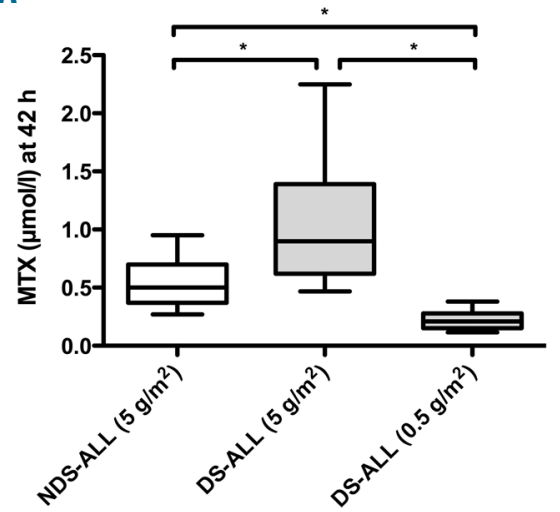

C

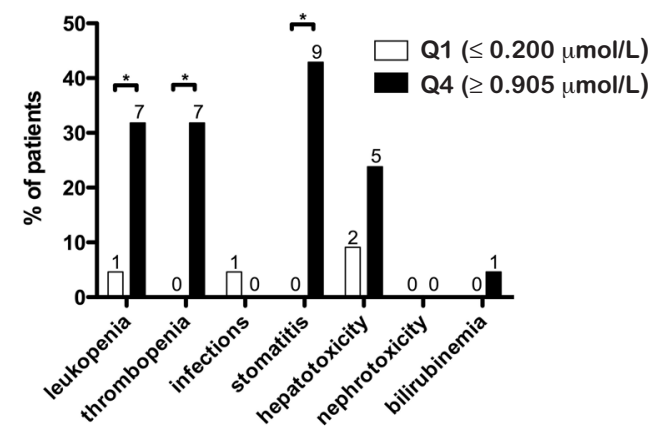

B

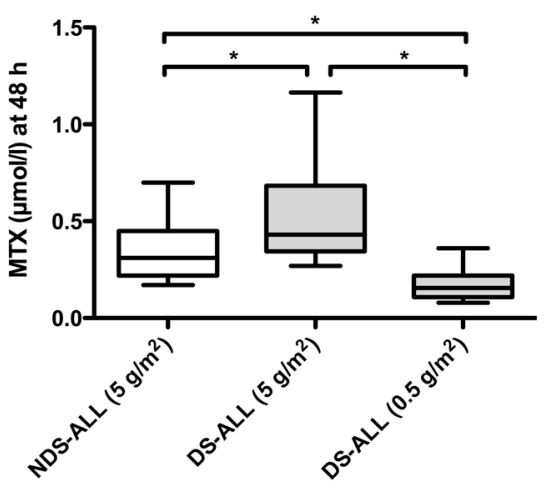

D

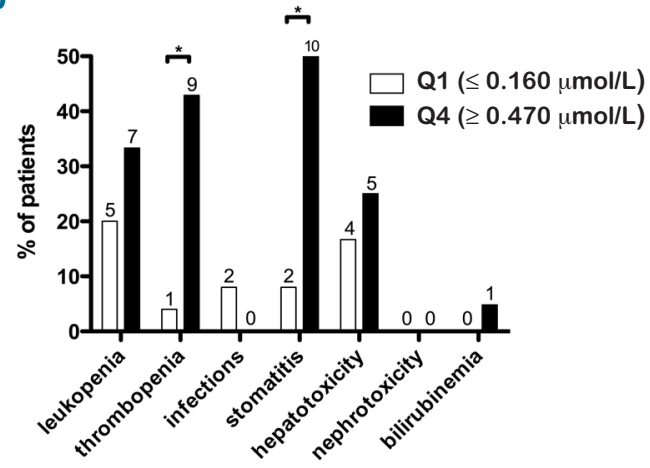

Figure 4. Methotrexate (MTX) plasma levels at 42 and 48 hours after the start of the first high dose MTX course and grade $3 / 4$ toxicities in Down syndrome acute lymphoblastic leukemia according to MTX plasma levels. (A) and B) MTX plasma levels at $42 \mathrm{~h}$ (A) and $48 \mathrm{~h}$ (B) after start of the first HD-MTX administration. MTX dosage subgroups are indicated. ${ }^{*} P \leq 0.05$, Mann-Whitney $U$ test. (C and D) Comparison of grade $3 / 4$ toxicities in DS-ALL according to MTX plasma level quartiles at 42 hours (C) and 48 hours (D) after the start of the first high dose MTX (HD-MTX) administration. Q1: first/lowest quartile; Q4: fourth/highest quartile; respective MTX plasma concentration is given in $\mu \mathrm{mol} / \mathrm{L}$. ${ }^{*} P \leq 0.05$, Fisher's exact test. The number on top of each bar represents the number of patients.

Goto et al. proposed a lower MTX dose $\left(<3.0 \mathrm{~g} / \mathrm{m}^{2}\right)$ for DS-ALL patients and possible treatment modifications with novel therapeutics considering the biology of DSALL, e.g. JAK2 inhibitors in those cases with JAK/STAT pathway activation. ${ }^{5}$ In addition, the use of the bispecific antibody blinatumomab might be beneficial to patients with DS-ALL. ${ }^{17}$

Of note, in contrast to published data recently summarized $^{18}$ none of the patients included in our study suffered from grade $3 / 4$ nephrotoxicity after the first HD-MTX course. There is no obvious explanation for this difference and we can only speculate that vigorous hydration and alkalization in our patients as described in detail in the protocol was sufficient to prevent MTX crystal precipitation in the kidneys and subsequent impairment of renal function. ${ }^{19,20}$ Moreover, cut-offs to define nephrotoxicity might differ between the studies.

Among the DS-ALL cohort we found a MTX plasma level-toxicity correlation as patients with high plasma levels at 42 and 48 hours after the start of the first MTX infusion showed higher rates of grade $3 / 4$ toxicities compared to patients within the lowest MTX plasma level quartile. This observation is consistent with another report showing higher rates of grade $3 / 4$ mucositis in pediatric patients with osteosarcoma that had higher median MTX plasma levels at 48 hours. In addition, MTX plasma levels were higher in DS-ALL compared to NDS-ALL when the same doses were given, which could partially explain the higher toxicity in DS-ALL. In contrast, Buitenkamp et al. observed neither higher MTX levels in DS-ALL compared to NDSALL nor any association between MTX area under the curve (AUC) and toxicity. ${ }^{8}$ The authors applied a case-control study approach and the number of DS-ALL patients was lower than this study. Moreover in this study, MTX AUC and not plasma levels were considered when looking at toxicity, and therefore the results are not directly comparable with ours. However, we agree with the conclusion made by Buitenkamp et al. that differences in pharmacodynamics could also significantly contribute to the higher MTX toxicity in DS-ALL patients. ${ }^{8}$ This hypothesis is supported by our observation that DS-ALL patients who showed lower MTX plasma levels after $0.5 \mathrm{~g} / \mathrm{m}^{2}$ MTX than NDS-ALL with $5 \mathrm{~g} / \mathrm{m}^{2}$ still experienced higher rates of toxicity after the first HD-MTX course.

Based on our data one might speculate that lower cut offs for forced diuresis and LCV rescue may reduce toxicities in DS-ALL patients. Furthermore, since DS-ALL had higher MTX plasma levels after receiving $5 \mathrm{~g} / \mathrm{m}^{2}$ MTX 
compared to NDS-ALL one could further argue that DSALL patients do not require MTX doses as high as $5 \mathrm{~g} / \mathrm{m}^{2}$ to get the same plasma levels and subsequent effect with respect to relapse prevention.

In an attempt to shed further light on MTX susceptibility in DS-ALL patients we genotyped the rs1051266 80G >A SNP of the folate and MTX transporter SLC19A1 on chromosome. ${ }^{21}$ The allele frequencies in our DS-ALL cohort are consistent with previous published frequencies in a healthy DS cohort. ${ }^{22}$ Since Baslund et al. found the A-variant of the transporter to be more effective in taking up fluorescence labeled MTX into lymphocytes of healthy euploide blood donors, ${ }^{11}$ we speculated that more MTX might be taken up into cells of DS-ALL patients that are homozygous for the presumably higher active A-variant (AAA) and that this may lead to higher rates of severe MTX-related toxicities compared to patients with the GGG allele combination. Thus, we hypothesized that genotyping of rs1051266 might be a diagnostic tool to predict the severity of toxicities in DS-ALL patients, but we were unable to confirm this hypothesis. However, pharmacokinetics of a single drug or the impact of a single SNP of a transporter in complex chemotherapy regimens may have limited power to explain the differences in effects and side effects. Other SNP in SLC19A1 and SNP in other genes of transporters or enzymes in the folate/MTX metabolism may also play a role. Therefore, further research including haplotype analysis, investigations of the effect of trisomy 21 on MTX metabolism by using transcriptomics or microarrays and the evaluation of SLC19A1 expression profile on mRNA and protein level as well as its transportation activity are needed. Moreover, 6-MP co-medication during consolidation should be considered as differences in DS patients with regards to 6-MP metabolism have been described. ${ }^{23}$ This might be important in HD-MTX consolidation but also in maintenance therapy, in which both MTX and 6-MP are administered since up to $40 \%$ of treatment-related deaths in DS-ALL occur during maintenance therapy. ${ }^{6,24}$

In conclusion, dose reduction in the first HD-MTX course of consolidation therapy led to a decrease of severe MTX-associated toxicities without increasing the risk of relapse for DS-ALL patients.

\section{Funding}

The authors would like to thank Deutsche Krebshilfe, Deutsche José Carreras Leukämie-Stiftung (DJCLS), Gesellschaft für Pädiatrische Onkologie und Hämatologie $(G P O H)$, José Carreras-GPOH Promotionsstipendium 03 PSG/2017 for financial support.

\section{References}

1. Hasle H, Friedman JM, Olsen JH, Rasmussen SA. Low risk of solid tumors in persons with Down syndrome. Genet Med. 2016;18(11): 1151-1157.

2. Dordelmann M, Schrappe M, Reiter A, et al. Down's syndrome in childhood acute lymphoblastic leukemia: clinical characteristics and treatment outcome in four consecutive BFM trials. Berlin-FrankfurtMünster Group. Leukemia. 1998;12(5): 645-651.

3. Whitlock JA. Down syndrome and acute lymphoblastic leukaemia. Br J Haematol. 2006;135(5):595-602.

4. Arico M, Ziino O, Valsecchi MG, et al. Acute lymphoblastic leukemia and Down syndrome. Cancer. 2008;113(3):515-521.

5. Goto $H$, Inukai $T$, Inoue $H$, et al. Acute lymphoblastic leukemia and Down syndrome: the collaborative study of the Tokyo Children"s Cancer Study Group and the Kyushu Yamaguchi Children"s Cancer Study Group. Int J Hematol. 2011; 93(2):192-198.

6. Buitenkamp TD, Izraeli S, Zimmermann $M$, et al. Acute lymphoblastic leukemia in children with Down syndrome: a retrospective analysis from the Ponte di Legno study group. Blood. 2014;123(1):70-77.

7. Taub JW, Ge Y. Down syndrome, drug metabolism and chromosome 21. Pediatr Blood Cancer. 2004;44(1):33-39.

8. Buitenkamp TD, Mathot RAA, de Haas V, Pieters R, Zwaan CM. Methotrexateinduced side effects are not due to differences in pharmacokinetics in children with Down syndrome and acute lymphoblastic leukemia. Haematologica. 2010;95(7):
1106-1113

9. Schmiegelow K. Advances in individual prediction of methotrexate toxicity: a review. Br J Haematol. 2009;146(5):489503.

10. Matherly LH, Wilson MR, Hou Z. The major facilitative folate transporters solute carrier 19A1 and solute carrier 46A1: biology and role in antifolate chemotherapy of cancer. Drug Metabolism and Disposition. 2014;42(4):632-649.

11. Baslund B, Gregers J, Nielsen CH. Reduced folate carrier polymorphism determines methotrexate uptake by B cells and CD4+ T cells. Rheumatology. 2007;47(4):451-453.

12. Whetstine JR, Gifford AJ, Witt T, et al. Single nucleotide polymorphisms in the human reduced folate carrier: characterization of a high-frequency G/A variant at position 80 and transport properties of the His(27) and $\operatorname{Arg}(27)$ carriers. Clin Cancer Res. 2001;7(11):3416-3422.

13. Radtke S, Zolk O, Renner B, et al. Germline genetic variations in methotrexate candidate genes are associated with pharmacokinetics, toxicity, and outcome in childhood acute lymphoblastic leukemia. Blood. 2013; 121(26):5145-5153.

14. Whitlock JA, Sather HN, Gaynon P, et al. Clinical characteristics and outcome of children with Down syndrome and acute lymphoblastic leukemia: a Children's Cancer Group study. Blood. 2005;106(13):4043-4049.

15. Shah N, Al-Ahmari A, Al-Yamani A, Dupuis L, Stephens D, Hitzler J. Outcome and toxicity of chemotherapy for acute lymphoblastic leukemia in children with down syndrome. Pediatr Blood Cancer. 2009;52(1):14-19.

16. Derouet A, Petit A, Baruchel A, et al.
Impact of therapy in a cohort of unselected children with Down Syndrome-associated Acute Lymphoblastic Leukaemia. Br J Haematol. 2016;174(6):983-985.

17. Wadhwa A, Kutny MA, Xavier AC. Blinatumomab activity in a patient with Down syndrome B-precursor acute lymphoblastic leukemia. Pediatr Blood Cancer. 2017;65(2):e26824.

18. Schmiegelow K, Müller K, Mogensen SS, et al. Non-infectious chemotherapy-associated acute toxicities during childhood acute lymphoblastic leukemia therapy. F1000Res. 2017;6:444.

19. Sand TE, Jacobsen S. Effect of urine $\mathrm{pH}$ and flow on renal clearance of methotrexate. Eur J Clin Pharmacol. 1981;19(6):453-456.

20. Garneau AP, Riopel J, Isenring P. Acute methotrexate-induced crystal nephropathy. N Engl J Med. 2015; 373(27):26912693.

21. Park JA, Shin HY. Influence of genetic polymorphisms in the folate pathway on toxicity after high-dose methotrexate treatment in pediatric osteosarcoma. Blood Res. 2016; 51(1):50-57.

22. Chango A, Willequet F, Fillon-Emery $\mathrm{N}$, Nicolas JP, Bléhaut $H$. The single nucleotide polymorphism (80G-->A) of reduced folate carrier gene in trisomy 21. Am J Clin Nutr. 2004:80(6):1667-1669.

23. Palle J, Frost BM, Britt-Marie F, et al Thioguanine pharmacokinetics in induction therapy of children with acute myeloid leukemia. Anti-Cancer Drugs. 2009;20(1):7-14.

24. O'Connor D, Bate J, Wade $\mathrm{R}$, et al. Infection-related mortality in children with acute lymphoblastic leukemia: an analysis of infectious deaths on UKALL2003. Blood. 2014;124(7): 1056-1061. 